\title{
Bell's theorem without inequalities and without unspeakable information
}

\author{
Adán Cabelld* \\ Departamento de Física Aplicada II, Universidad de Sevilla, 41012 Sevilla, Spain
}

(Dated: October 4, 2018)

\begin{abstract}
A proof of Bell's theorem without inequalities is presented in which distant local setups do not need to be aligned, since the required perfect correlations are achieved for any local rotation of the local setups.
\end{abstract}

\section{INTRODUCTION}

For the last 10 years, Asher has always been right there to help me with his advice and knowledge. He has always been reachable by e-mail to give a quick and precise answer to all my queries with a patience and enthusiasm far beyond that required by common courtesy between scientists. It is therefore an honor for me to be here as a friend to celebrate his 70th birthday. For this occasion I have chosen to speak about a recurring subject in our correspondence, namely, Bell's theorem, in light of one of Asher's observations, that is to say, that the sharing of reference frames between distant observers is an expensive resource.

Usual proofs of Bell's theorem assume that the distant observers who perform spacelike separated measurements share a common reference frame so that they can prepare and measure spin components relative to it. However, a common reference frame is not a free preexisting element in a communication scenario. Establishing a perfect alignment between local reference frames requires the transmission of an infinite amount of information. Therefore, a common reference frame should be considered an expensive resource. Indeed, the search for optimal strategies to establish a common direction or Cartesian frame between distant observers to any desired accuracy has attracted much interest [1, 2, 3, 4, 5, 6].

Addressing the question of whether or not it is possible to develop a proof of Bell's theorem without assuming a common reference frame seems to be relevant from a different point of view. In 1988 Yuval Ne'eman argued that the answer to the puzzle posed by Bell's theorem was to be found in the implicit assumption that the detectors were aligned. Ne'eman apparently believed that the two detectors were connected through the space-time affine connection of general relativity 7].

However, from a practical point of view, that is, for an experiment to show the violation of a Bell's inequality, the fact that a perfect alignment between the source of the entangled states and the setups of the distant measurements is difficult to achieve is not particularly relevant, since perfect alignment is not essential for such a test, because a violation can be obtained even with an approximate alignment. However, in the so-called proofs of Bell's theorem "without inequalities" [9, 10, 11, 12, 13, 14] perfect alignment seems to be essential. These proofs are based on inferences motivated by Einstein-PodolskyRosen's (EPR's) condition for elements of reality:
"If, without in any way disturbing a system, we can predict with certainty (i.e., with a probability equal to unity) the value of a phys- ical quantity, then there exist an element of physical reality corresponding to this physical quantity" [8].

These inferences arrive at a contradiction. In these proofs, any imperfection in the required alignments leads to the disappearance of the required inferences.

The term "unspeakable information" was coined by Peres and Scudo 6] to designate information that cannot be represented by a sequence of discrete symbols, such as a direction in space or a reference frame. Here we shall show that there is a method to prove Bell's theorem without inequalities without it being necessary that the observers share unspeakable information or, more precisely, without distant local setups needing to be aligned.

The proof is based on the fact that the required perfect correlations occur for any local rotation of the local setups. Therefore, we shall show not only that the apparently innocent EPR's elements of reality are incompatible with quantum mechanics, but even that an apparently more innocent criterion for elements of reality, namely,

\begin{abstract}
"If, without in any way disturbing a system, we can predict with certainty (i.e., with a probability equal to unity) the value of a physical quantity, independently of the orientation of the measurement apparatus used, then there exist an element of physical reality corresponding to this physical quantity",
\end{abstract}

is also incompatible with quantum mechanics.

\section{PROOF}

Let us consider a source emitting systems of eight qubits prepared in the state

$$
|\eta\rangle=\left(\left|\phi_{0} \phi_{0}\right\rangle+\sqrt{3}\left|\phi_{0} \phi_{1}\right\rangle+\sqrt{3}\left|\phi_{1} \phi_{0}\right\rangle\right) / \sqrt{7},
$$

where $\left|\phi_{0}\right\rangle$ and $\left|\phi_{1}\right\rangle$ are the two singlet states obtained adding up four spin- $\frac{1}{2}$ momenta,

$$
\left|\phi_{0}\right\rangle=\frac{1}{2}(|0101\rangle-|0110\rangle-|1001\rangle+|1010\rangle),
$$




$$
\begin{aligned}
\left|\phi_{1}\right\rangle= & \frac{1}{2 \sqrt{3}}(2|0011\rangle-|0101\rangle-|0110\rangle-|1001\rangle \\
& -|1010\rangle+2|1100\rangle) .
\end{aligned}
$$

These states were introduced by Kempe et al. in the context of decoherence-free fault-tolerant universal quantum computation [15]. Let us suppose that the first four qubits prepared in $|\eta\rangle$ fly to Alice and the second four qubits fly to a distant observer, Bob. On her/his four qubits, each observer randomly chooses to measure either $F$ or $G$, defined as

$$
\begin{aligned}
& F=-\left|\phi_{0}\right\rangle\left\langle\phi_{0}|+| \phi_{1}\right\rangle\left\langle\phi_{1}\right|, \\
& G=-\left|\psi_{0}\right\rangle\left\langle\psi_{0}|+| \psi_{1}\right\rangle\left\langle\psi_{1}\right|,
\end{aligned}
$$

where $\left|\psi_{0}\right\rangle$ and $\left|\psi_{1}\right\rangle$ are obtained, respectively, from $\left|\phi_{0}\right\rangle$ and $\left|\phi_{1}\right\rangle$, by permuting qubits 2 and 3, i.e.,

$$
\begin{aligned}
\left|\psi_{0}\right\rangle= & \frac{1}{2}(|0011\rangle-|0110\rangle-|1001\rangle+|1100\rangle) \\
= & \frac{1}{2}\left(\left|\phi_{0}\right\rangle+\sqrt{3}\left|\phi_{1}\right\rangle\right), \\
\left|\psi_{1}\right\rangle= & \frac{1}{2 \sqrt{3}}(-|0011\rangle+2|0101\rangle-|0110\rangle-|1001\rangle \\
& +2|1010\rangle-|1100\rangle) \\
= & \frac{1}{2}\left(\sqrt{3}\left|\phi_{0}\right\rangle-\left|\phi_{1}\right\rangle\right) .
\end{aligned}
$$

The observable $F(G)$ has three possible outcomes: -1 , corresponding to $\left|\phi_{0}\right\rangle\left(\left|\psi_{0}\right\rangle\right), 1$ corresponding to $\left|\phi_{1}\right\rangle$ $\left(\left|\psi_{1}\right\rangle\right)$, and 0 , which never occurs because the local subsystems have total spin zero. Measuring $F$ is thus equivalent to distinguishing with certainty between $\left|\phi_{0}\right\rangle$ and $\left|\phi_{1}\right\rangle$ with a single test on the four qubits, and measuring $G$ is equivalent to distinguishing with certainty between $\left|\psi_{0}\right\rangle$ and $\left|\psi_{1}\right\rangle$. Alice's measurements on qubits 1 to 4 are assumed to be spacelike separated from Bob's measurements on qubits 5 to 8 .

The state $|\eta\rangle$ can also be expressed as

$$
\begin{aligned}
|\eta\rangle= & \left(4\left|\phi_{0} \psi_{0}\right\rangle+\sqrt{3}\left|\phi_{1} \psi_{0}\right\rangle+3\left|\phi_{1} \psi_{1}\right\rangle\right) / 2 \sqrt{7} \\
= & \left(4\left|\psi_{0} \phi_{0}\right\rangle+\sqrt{3}\left|\psi_{0} \phi_{1}\right\rangle+3\left|\psi_{1} \phi_{1}\right\rangle\right) / 2 \sqrt{7} \\
= & \left(7\left|\psi_{0} \psi_{0}\right\rangle+3 \sqrt{3}\left|\psi_{0} \psi_{1}\right\rangle+3 \sqrt{3}\left|\psi_{1} \psi_{0}\right\rangle\right. \\
& \left.-3\left|\psi_{1} \psi_{1}\right\rangle\right) / 4 \sqrt{7} .
\end{aligned}
$$

Moreover, since $\left|\phi_{0}\right\rangle,\left|\phi_{1}\right\rangle,\left|\psi_{0}\right\rangle$, and $\left|\psi_{1}\right\rangle$ are invariant under the tensor product of four equal unitary operators, then they are invariant under local rotations. Therefore, expressions (11) and (8)-(10) remain unchanged after local rotations. Consequently, if $R_{A}$ and $\mathcal{R}_{A}\left(R_{B}\right.$ and $\left.\mathcal{R}_{B}\right)$ are rotations of Alice's (Bob's) setups for measuring, respectively, $F$ and $G$ relative to the reference frame of the source then, in the state $|\eta\rangle$, for any rotations $R_{A}, \mathcal{R}_{A}$, $R_{B}$, and $\mathcal{R}_{B}$,

$$
P\left(R_{A} F=1, R_{B} F=1\right)=0,
$$

$$
\begin{aligned}
& P\left(R_{A} F=1 \mid \mathcal{R}_{B} G=1\right)=1, \\
& P\left(R_{B} F=1 \mid \mathcal{R}_{A} G=1\right)=1, \\
& P\left(\mathcal{R}_{A} G=1, \mathcal{R}_{B} G=1\right)=\frac{9}{112},
\end{aligned}
$$

where $P\left(R_{A} F=1, R_{B} F=1\right)$ is the joint probability that both Alice and Bob obtain the outcome 1 when both perform experiment $F$ (or any experiment consisting on independently rotating their setups for measuring $F)$, and $P\left(R_{A} F=1 \mid \mathcal{R}_{B} G=1\right)$ is the probability that Alice obtains the outcome 1 when she performs experiment $F$ (or any experiment consisting on rotating her setup for measuring $F$ ), conditioned to Bob obtaining the outcome 1 when he performs experiment $G$ (or any experiment consisting on rotating his setup for measuring $G$ ).

From property (14), if both Alice and Bob choose the setup for measuring $G$, then in $8 \%$ of the events the outcome is 1 in both cases. This is true even if Alice applies any rotation $\mathcal{R}_{A}$ to her setup and Bob applies any rotation $\mathcal{R}_{B}$ to his setup.

From property (13), if Alice measures $G$ and obtains the outcome 1 , then she can predict with certainty that, if Bob measures $F$, he will obtain 1. According to Einstein, Podolsky, and Rosen (EPR), this fact must be interpreted as sufficient evidence that there is a local "element of reality" in Bob's qubits determining this outcome [8]. Moreover, EPR reasoning seems to be even more inescapable in our example, since Alice's prediction with certainty is valid even if Alice applies any rotation $\mathcal{R}_{A}$ to her setup for measuring $G$ and Bob applies any rotation $R_{B}$ to his setup for measuring $F$.

Analogously, from property (12), if Bob measures $G$ (or $\mathcal{R}_{A} G$ ) and obtains 1 , then he can predict with certainty that, if Alice measures $F$ (or $R_{A} F$ ), she will obtain 1. Again, according to EPR, there must be a local element of reality in Alice's qubits determining this outcome.

Therefore, assuming EPR's point of view, for at least $8 \%$ of the systems prepared in the state $|\eta\rangle$, there must be two joint local elements of reality: one for $\mathrm{Al}-$ ice's qubits, corresponding to $R_{A} F=1$, and one for Bob's qubits, corresponding to $R_{B} F=1$. However, this inference is in contradiction with property (11), which states that the joint probability of obtaining the outcomes $R_{A} F=1$ and $R_{B} F=1$ is zero. The logical structure of the proof is summarized in Fig. [1]

This is a simple and powerful proof that the concept of element of reality, as defined by EPR, is incompatible with quantum mechanics, even if the predictions with certainty are valid not only for a particular alignment of the distant setups but for any possible rotation. 


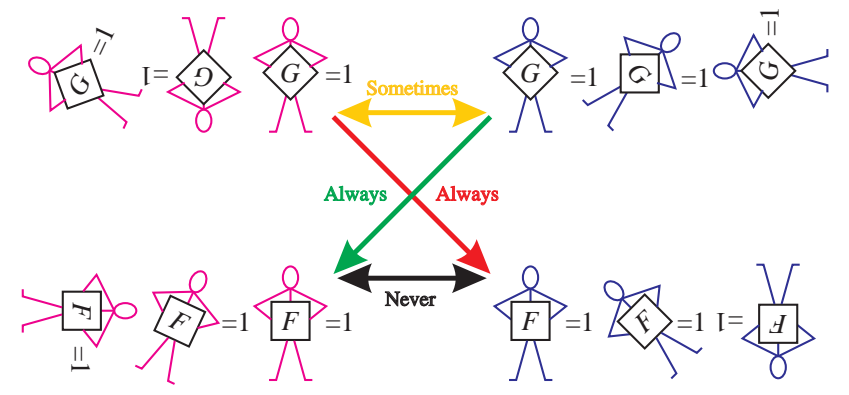

FIG. 1: Let us suppose that Alice (up in the left-hand side) measures $G$ and obtains the result 1 . Then, if Bob (down in the right-hand side), who is spacelike separated from Alice, measures $F$, he always obtains 1 , even if she/he rotates her/his measurement apparatus. Analogously, if Bob (up in the right-hand side) measures $G$ and obtains 1 , then he can predict that, if Alice (down in the left-hand side) measures $F$, she always obtains 1 , even if Alice/Bob rotates her/his apparatus. If Alice and Bob measure $G$, sometimes (in $8 \%$ of the cases) they both obtain 1 . In those cases, what if, instead of measuring $G$, they had measured $F$ ?. If EPR's elements of reality do exist, then, at least in $8 \%$ of the cases, both of them would have obtained $F=1$. However, they never both obtain 1.

\section{REMARKS}

The fact that the required perfect correlations occur for any local rotation of the local setups arises from the fact that the state $|\eta\rangle$ is invariant under local rotations,

$$
R_{A} \otimes R_{B}|\eta\rangle=|\eta\rangle
$$

This property comes from the fact that the states $\left|\phi_{0}\right\rangle$ and $\left|\phi_{1}\right\rangle$ are invariant under the same unitary transformation applied to the four qubits,

$$
U \otimes U \otimes U \otimes U\left|\phi_{j}\right\rangle=\left|\phi_{j}\right\rangle
$$

where $U$ is any unitary operation on one qubit. This invariance of the four-qubit states $\left|\phi_{0}\right\rangle$ and $\left|\phi_{1}\right\rangle$ has been recently demonstrated in a laboratory using four-photon polarization-entangled states produced by spontaneous parametric down-conversion [16].

A practical advantage and a remarkable property of this proof is that measuring $F$ or $G$ does not require collective measurements on two or more qubits but instead a single test on each of the four qubits. Measuring $F$ is equivalent to distinguishing between $\left|\phi_{0}\right\rangle$ and $\left|\phi_{1}\right\rangle$ with a single test. Remarkably, the only two orthogonal states invariant under any tensor product of four equal unitary operators that can be reliably distinguished by fixed measurements on the four individual qubits are $\left|\phi_{0}\right\rangle$ and $\left|\phi_{1}\right\rangle$ and those obtained from them by permuting qubits (such as $\left|\psi_{0}\right\rangle$ and $\left.\left|\psi_{1}\right\rangle\right)$ [17].

To distinguish with certainty between $\left|\phi_{0}\right\rangle$ and $\left|\phi_{1}\right\rangle$, it is enough to measure the spin component of the first two qubits along the same direction and the spin component of the other two qubits along a perpendicular direction. This can be seen by resorting to the invariance under any tensor product of four equal unitary operators and expressing these states in the basis of eigenstates of $\sigma_{z 1} \otimes$ $\sigma_{z 2} \otimes \sigma_{x 3} \otimes \sigma_{x 4}$,

$$
\begin{aligned}
\left|\phi_{0}\right\rangle= & \frac{1}{2}(-|01 \overline{0} \overline{1}\rangle+|01 \overline{1} \overline{0}\rangle+|10 \overline{0} \overline{1}\rangle-|10 \overline{1} \overline{0}\rangle), \\
\left|\phi_{1}\right\rangle= & \frac{1}{2 \sqrt{3}}(|00 \overline{0} \overline{0}\rangle-|00 \overline{0} \overline{1}\rangle-|00 \overline{1} \overline{0}\rangle+|00 \overline{1} \overline{1}\rangle \\
& -|01 \overline{0} \overline{0}\rangle+|01 \overline{1} \overline{1}\rangle-|10 \overline{0} \overline{0}\rangle+|10 \overline{1} \overline{1}\rangle \\
& +|11 \overline{0} \overline{0}\rangle+|11 \overline{0} \overline{1}\rangle+|11 \overline{1} \overline{0}\rangle+|11 \overline{1} \overline{1}\rangle),
\end{aligned}
$$

where $\sigma_{z}|0\rangle=|0\rangle, \sigma_{z}|1\rangle=-|1\rangle, \sigma_{x}|\overline{0}\rangle=|\overline{0}\rangle, \sigma_{x}|\overline{1}\rangle=$ $-|\overline{1}\rangle[|\overline{0}\rangle=(|0\rangle+|1\rangle) / \sqrt{2}$ and $|\overline{1}\rangle=(|0\rangle-|1\rangle) / \sqrt{2}]$. According to (17) and (18), if the measurements on the individual qubits are $\sigma_{z 1}, \sigma_{z 2}, \sigma_{x 3}, \sigma_{x 4}$ (or any rotation thereof), then, among the 16 possible outcomes, four occur (with equal probability) only in the state $\left|\phi_{0}\right\rangle$, and the other twelve occur (with equal probability) only in the state $\left|\phi_{1}\right\rangle$ (this has been experimentally demonstrated in [16]). Therefore, to measure $F(G)$, it is enough to measure the spin component of qubits 1 and 2 (1 and 3 ) along the same direction and the spin component of the other two qubits along a perpendicular direction.

On the other hand, fixed measurements on the four individual qubits are not enough for a GreenbergerHorne-Zeilinger-like proof without unspeakable information. Such a proof requires conditioned measurements on the four individual qubits [18].

\section{CONCLUSIONS}

What conclusions should we draw from all this? David Mermin would say:

"Some people conclude from all this that the character of your [or Alice's] stuff can indeed be altered by decisions made by your far away friend [Bob]. This is called quantum nonlocality. Others, myself among them, conclude that it is treacherous to make judgments about the character of your stuff, and extremely treacherous to reason from what actually happened to what might have happened but didn't" [19].

Which is a less concise way of saying what Asher perfectly summarized 26 years ago:

"Unperformed experiments have no results" [20].

\section{ACKNOWLEDGEMENTS}

The author thanks the organizers of the conference $R e$ cent Developments in Quantum Physics, specially Ady 
Mann and Liz Youdim, and the Spanish Ministerio de Ciencia y Tecnología Project BFM2002-02815 for support.

* Electronic address: adan@us.es

[1] A. Peres and P.F. Scudo, Phys. Rev. Lett. 86, 4160 (2001).

[2] A. Peres and P.F. Scudo, Phys. Rev. Lett. 87, 167901 (2001).

[3] E. Bagan, M. Baig, A. Brey, R. Muñoz-Tapia, and R. Tarrach, Phys. Rev. A 63, 052309 (2001).

[4] E. Bagan, M. Baig, and R. Muñoz-Tapia, Phys. Rev. A 64, 022305 (2001).

[5] E. Bagan, M. Baig, and R. Muñoz-Tapia, Phys. Rev. Lett. 87, 257903 (2001).

[6] A. Peres and P.F. Scudo, in Quantum Theory: Reconsideration of Foundations, edited by A. Khrennikov (Växjö University Press, Växjö, Sweden, 2002).
[7] N.D. Mermin (private communication).

[8] A. Einstein, B. Podolsky, and N. Rosen, Phys. Rev. 47, 777 (1935).

[9] D.M. Greenberger, M.A. Horne, and A. Zeilinger, in Bell's Theorem, Quantum Theory, and Conceptions of the Universe, edited by M. Kafatos (Kluwer, Dordrecht, 1989), p. 69.

[10] N.D. Mermin, Phys. Rev. Lett. 65, 3373 (1990).

[11] L. Hardy, Phys. Rev. Lett. 68, 2981 (1992).

[12] L. Hardy, Phys. Rev. Lett. 71, 1665 (1993).

[13] A. Cabello, Phys. Rev. Lett. 86, 1911 (2001).

[14] A. Cabello, Phys. Rev. Lett. 87, 010403 (2001).

[15] J. Kempe, D. Bacon, D.A. Lidar, and K.B. Whaley, Phys. Rev. A 63, 042307 (2001).

[16] M. Bourennane, M. Eibl, S. Gaertner, C. Kurtsiefer, A. Cabello, and H. Weinfurter, e-print quant-ph/0309041 (to be published in Phys. Rev. Lett.).

[17] A. Cabello, Phys. Rev. Lett. 91, 230403 (2003).

[18] A. Cabello, Phys. Rev. A 68, 042104 (2003).

[19] N.D. Mermin, Am. J. Phys. 71, 296 (2003).

[20] A. Peres, Am. J. Phys. 46, 745 (1978). 